\title{
ESTUDIO COMPARATIVO DEL USO DE PARTÍCULAS DISCURSIVAS EN DOS TIPOS DE HABLA INFORMAL
}

\author{
Comparative study about the use of discursive particles in two types of informal speech
}

\section{María Benavides González*}

\author{
David Pérez Retana**
}

\begin{abstract}
RESUMEN
En este artículo, se describen y se comparan once partículas discursivas utilizadas en dos tipos de habla informal: el habla oral y el habla escrita en la red social Facebook, con el fin de adentrarnos en la discusión sobre la influencia de la oralidad en el lenguaje escrito en Internet. El análisis considera, principalmente, la función de la partícula discursiva elegida, esta se ejemplifica en su uso en contexto; luego, dependiendo de la modalidad, se establecen similitudes y diferencias de uso, las cuales nos permiten identificar las características particulares de una modalidad discursiva mixta, como lo es la escritura en medios electrónicos. Palabras clave: ciberhabla, habla informal, partículas discursivas, mecanismos de cohesión, discurso oral y escrito.
\end{abstract}

\section{ABSTRACT}

This article describes and compares eleven discursive particles used in two types of informal speech: the oral speech and the written in the social network Facebook. The purpose of this is to get into the discussion of the influence of orality in the language in Internet. The function of those chosen discursive particles is considered in this analysis and is exemplified by its context. Also, similarities and differences are established to identify the particular characteristics of a mixed discursive mode, as is the writing on electronic media.

Key Words: Netspeak, informal speaking, discourse particles, cohesive mechanism, speech and writing. 


\section{Introducción}

Cuando escuchamos hablar sobre el estilo de habla informal, existe una tendencia a vincularlo con el discurso oral espontáneo; no obstante, con el desarrollo de las tecnologías de la información, han ido apareciendo en escena nuevas plataformas de comunicación que privilegian un estilo escrito informal, tal es el caso de los chats, los mensajes de texto telefónicos o las conversaciones en redes sociales. Ante esta realidad, surgió el deseo de comparar ambos tipos de habla informal: la oral y la utilizada en redes sociales, con el propósito de adentrarnos en una discusión que parece preocupar a muchos estudiosos del lenguaje en Internet: ies este tipo de discurso simplemente una oralización de la escritura?

Para responder a esta interrogante, hemos decidido efectuar un análisis comparativo sobre el empleo de las partículas discursivas en estas dos modalidades. Claramente, aunque no se trata de un estudio exhaustivo, más bien descriptivo, sus resultados nos pueden brindar pistas importantes sobre los puntos de (des) encuentro entre ambas modalidades, al conocer cómo emplean los hablantes dichas partículas en la construcción de sus discursos.

Tal estudio se deriva de los resultados obtenidos de dos investigaciones independientes: Los marcadores discursivos en el español informal en Costa Rica: una propuesta de diccionario (Benavides, 2014) y Los mecanismos de cohesión textual en las conversaciones de Facebook (Pérez, 2014). En la primera, se describen y analizan las funciones de las partículas discursivas, y se señala su significado, el que se obtiene principalmente del contexto y de las relaciones establecidas en el discurso. A partir de esta información, la autora propuso la construcción de un diccionario activo (Portolés, 2001), cuyas definiciones son de tipo funcional (Porto Dapena, 2002). Para el tema que nos ocupa, se consideran los usos indicados para los elementos escogidos.

En la segunda investigación, se estudian los mecanismos de cohesión textual empleados por usuarios de Facebook en sus conversaciones.
El análisis se efectúa con base en los postulados teóricos de la lingüística textual de Beaugrande y Dressler (1997). Específicamente, se analiza el empleo de las partículas discursivas, las formas de referencia, la elipsis y los mecanismos léxicos de cohesión (sinonimia, repetición, repetición parcial, paralelismo y paráfrasis).

\section{Marco teórico}

\subsection{Partículas discursivas}

Es necesario distinguir los términos partícula discursiva y marcador discursivo; ambos se emplean en las investigaciones indicadas anteriormente, no obstante, para este artículo, se utilizará el primero de ellos. Es importante recalcar que este tipo de palabras recibe diversas denominaciones, de acuerdo con la perspectiva de investigación seleccionada (por ejemplo: conectores, conectivos, operadores, enlaces, nexos, elementos relacionales, apéndice, marcadores discursivos). Para este artículo, tal como se mencionó, hemos decidido utilizar "partícula discursiva", pues se trata de elementos lingüísticos que guían la interpretación del discurso, es decir, tienen un carácter más procedimental que conceptual (Briz, Pons y Portolés, 2008). Las formas que consideramos comparten, en general, la característica de que el valor de la partícula está ligado a la interacción.

Por otra parte, siguiendo a Garcés (2008: 15), "partícula" es un concepto más abarcador referido a cualquier palabra invariable o locución que guíe, por su significado, el procesamiento de otra unidad con significado conceptual; a diferencia de "marcador discursivo", que alude al nivel en el cual se inserta su descripción y pone de relieve su significado específico, que consiste en proporcionar instrucciones para la correcta interpretación de los enunciados.

\subsection{Tipos de partículas discursivas}

A continuación, definimos las partículas discursivas elegidas para esta comparación, tal como se expone en Benavides (2014), y siguiendo los criterios indicados por Portolés (2001): 
a. Conectores: vinculan semántica y pragmáticamente un miembro del discurso con otro miembro anterior, o con una posición fácilmente accesible.

i. Conector aditivo: además. Une a un miembro discursivo anterior a otro con la misma orientación argumentativa.

ii. Conector consecutivo: entonces. Presenta el miembro del discurso en el que se encuentran como una consecuencia de un miembro anterior.

b. Reformuladores: Presentan el miembro del discurso en el que se encuentran como una nueva formulación de lo que se pretendió decir con un miembro anterior.

i. Reformulador explicativo: es decir (que), o sea, es que. Presentan el miembro del discurso que introducen como una reformulación que aclara o explica lo que se ha querido decir con otro miembro anterior que pudiera ser poco comprensible.

ii. Reformulador rectificativo: más bien. Sustituye una primera formulación, que se presenta como incorrecta, por otra que la corrige o la mejora.

iii. Reformulador de recapitulación: al final. Presenta su miembro del discurso como una conclusión o recapitulación a partir de un miembro anterior o una serie de ellos.

c. Operadores discursivos: Cumplen la función de condicionar las posibilidades discursivas del miembro del discurso en el que se incluyen o al que afectan, pero sin relacionarlo, por su significado, con otro miembro anterior.

i. Operador de refuerzo argumentativo: claro, de hecho, en realidad, bueno, diay. $\mathrm{Su}$ significado refuerza como argumento el miembro del discurso en el que se encuentra frente a otros posibles argumentos, sean estos explícitos o implícitos.

Otras categorías empleadas por Pérez (2014) corresponden a:

d. Reactivo de desacuerdo (Calsamiglia y Tusón, 1999): bueno, diay. Conectores que cumplen una función reactiva, es decir, expresan una reacción negativa ante el contenido que transmiten.

e. Estructuradores de la información: Señalan la organización informativa de los discursos, carecen de significado argumentativo.

i. Comentadores (Martín Zorraquino y Portolés, 1999): bueno, diay. Introducen un nuevo comentario, lo que marca una distinción con el discurso previo.

\subsection{Habla informal}

Al referirnos al habla informal, se toma en consideración lo señalado por Briz (Briz y Val.es.co, 2000: 52): que es la conversación coloquial en la cual se presenta mayor relación de proximidad entre los participantes, así como de saber compartido y de cotidianidad; también, cuenta con menor grado de planificación y mayor finalidad interpersonal (es el tipo de características presentes, por ejemplo, en una conversación entre amigos, hablando de un tema cotidiano en un bar).

\section{Metodología}

\subsection{Recopilación de datos}

Los datos recopilados para la investigación de Benavides (2014) se tomaron de una muestra de 19 grabaciones en las que se incluyen debates y conversaciones con familiares y amigos; el requisito primordial que debían cumplir la 
situaciones de conversación es que participaran más de dos personas mayores de edad y que habitaran en el Valle Central de nuestro país. Las partículas discursivas analizadas se identificaron mediante la comparación con estudios sobre los marcadores en el habla costarricense (Solano Rojas, 1989, 1990, 1991; Castro Corrales, 2004; Sánchez Avendaño, 2005; Hernández, 2012) y por cumplir una serie de rasgos prosódicos, morfosintácticos, semánticos y pragmáticos ${ }^{1}$. Se registraron 25 marcadores discursivos, aun con una sola aparición; se describieron sus funciones $\mathrm{y}$, a partir de esto, se construyeron sus definiciones.

En el caso de la investigación de Pérez (2014), los datos fueron tomados de 30 conversaciones recopiladas en Facebook. $\mathrm{Se}$ trata de conversaciones informales entre "amigos", dado que tales textos son los más representativos de esta red social. Las interacciones fueron obtenidas del "muro" público de los usuarios. Asimismo, se procuró que los usuarios participantes en las interacciones fueran costarricenses, con el fin de crear un corpus más homogéneo en cuanto a las características diatópicas del español utilizado en las conversaciones. En cuanto a los temas de conversación no hubo restricciones; sin embargo, se buscó la variedad temática y se privilegiaron aquellas que fueran informales.

\subsection{Criterios para la transcripción del corpus}

Debido a la naturaleza diversa de los corpus utilizados en las investigaciones de Pérez (2014) y Benavides (2014), describimos los principales elementos que se consideraron para su transcripción.

En el corpus correspondiente al habla informal, se identificaron, para cada discurso (19 en total), los participantes, ya fuera por el número de interventor, el cargo o función que desempeña (por ejemplo: Mujer 1, Informante, Dependiente); las partículas discursivas se resaltan con negrita, otras partículas que se encuentran en el mismo enunciado, con cursiva.
Las muestras en este artículo contienen, también, otros símbolos tomados y adaptados del sistema de transcripción propuesto por Briz y Grupo Val.Es.Co (2000), a saber:

/ Pausa corta, inferior a un segundo

// Pausa larga, mayor o igual a un segundo

I/I Pausa de un segundo o más

$\S \quad$ Sucesión inmediata, sin pausa apreciable, entre dos emisiones de distintos hablantes

¿? Interrogaciones

¡! Exclamaciones

[ Lugar donde se inicia un solapamiento o superposición

] Final del habla simultánea

aa Alargamientos vocálicos

nn Alargamientos consonánticos

$\mathrm{pa}^{\prime} l \quad$ Fenómenos de fonética sintáctica entre palabras, especialmente marcados o que puedan dificultar la lectura.

(ininteligible) Fragmento indescifrable

Letra cursiva Reproducción e imitación de emisiones. Estilo directo, característico de los denominados relatos conversacionales.

En lo concerniente al corpus de Pérez (2014), las interacciones se transcribieron respetando la forma original en que fueron escritas por los participantes; por consiguiente, no se realizaron correcciones ortográficas ni gramaticales en la redacción de sus intervenciones. El único cambio aplicado a los textos corresponde al empleo de la negrita para resaltar la partícula analizada.

\subsection{Criterios para la clasificación}

La clasificación y la descripción del significado de las partículas responden a la expuesta por Portolés $(2000,2001)$ y Martín Zorraquino y Portolés (1999), la cual se presenta en el siguiente cuadro: 


\section{CUADRO 1.}

Clasificación de marcadores discursivos, según Martín Zorraquino y Portolés

\begin{tabular}{|c|c|c|}
\hline Estructuradores de la información & $\begin{array}{l}\cdot \text { Comentadores } \\
\cdot \text { Ordenadores } \\
\cdot \text { Digresores }\end{array}$ & $\begin{array}{l}\text { - Pues, pues bien, así las } \\
\text { cosas, etc. } \\
\cdot \text { En primer lugar/ en } \\
\text { segundo lugar/ por una } \\
\text { parte/por otra parte/ de un } \\
\text { lado/ de otro lado } \\
\text { - Por cierto, a todo esto, a } \\
\text { propósito de }\end{array}$ \\
\hline Conectores & $\begin{array}{l}\cdot \text { Conectores aditivos } \\
\cdot \text { Conectores consecutivos } \\
\cdot \text { Conectores contraargumentativos }\end{array}$ & $\begin{array}{l}\text { - Además, encima, aparte, } \\
\text { incluso, etc. } \\
\text { - Por tanto, por consiguiente, } \\
\text { por ende, en consecuencia, } \\
\text { de ahí, entonces, pues, así, } \\
\text { así pues, etc. } \\
\text { - En cambio, por el contrario, } \\
\text { antes bien, sin embargo, no } \\
\text { obstante, con todo, etc. }\end{array}$ \\
\hline Reformuladores & $\begin{array}{l}\text { - Reformuladores explicativos } \\
\text { - Reformuladores de rectificación } \\
\text { - Reformuladores de distanciamiento } \\
\text { - Reformuladores recapitulativos }\end{array}$ & $\begin{array}{l}\text { O sea, es decir, esto es, a } \\
\text { saber, etc. } \\
\text { - Mejor dicho, mejor aún, } \\
\text { más bien, etc. } \\
\text { - En cualquier caso, en todo } \\
\text { caso, de todos modos, etc. } \\
\text { - En suma, en conclusión, en } \\
\text { definitiva, en fin, al fin y al } \\
\text { cabo, etc. }\end{array}$ \\
\hline Operadores argumentativos ${ }^{2}$ hey & $\begin{array}{l}\text { - Operadores de refuerzo argumentativo } \\
\text { - Operadores de concreción }\end{array}$ & $\begin{array}{l}\text { - En realidad, en el fondo, de } \\
\text { hecho, etc. } \\
\text { - Por ejemplo, en particular, } \\
\text { etc. }\end{array}$ \\
\hline Marcadores conversacionales & $\begin{array}{l}\cdot \text { De modalidad epistémica } \\
\cdot \text { De modalidad deóntica } \\
\cdot \text { Enfocadores de la alteridad } \\
\cdot \text { Metadiscursivos conversacionales }\end{array}$ & $\begin{array}{l}\text { Claro, desde luego, por lo } \\
\text { visto. } \\
\cdot \text { Bueno, bien, vale, etc. } \\
\text { - Hombre, mira, oye, etc. } \\
\text { - Bueno, eh, este, etc. }\end{array}$ \\
\hline
\end{tabular}

Fuente: Martín Zorraquino y Portolés (1999: 4081-4082) 
Según se indicó en el apartado anterior, también se empleará la categoría Reactivo de desacuerdo de Calsamiglia y Tusón (1999), para identificar algunas partículas del corpus de Facebook.

Para el presente artículo, se seleccionaron las partículas registradas en ambos corpus y que, además, fueron analizadas de acuerdo con su función discursiva, de tal modo que permitiera un análisis comparativo por pares iguales. Se presentará la partícula, se compararán las funciones y se ejemplificará según corresponda. Los ejemplos son representativos y se organizaron de la siguiente manera: A, para aquellos extraídos del corpus de Facebook o discurso escrito; B, para los del discurso oral; entre paréntesis se numera cada uno de los ejemplos.

La lista de partículas elegidas es: además, entonces, o sea, más bien, claro, de hecho, en realidad, al final, bueno, diay, es que; se resaltan en negrita dentro de los ejemplos.

\section{Análisis de datos}

\section{Además}

Conector aditivo

A. (1) "Además, el tránsito hoy estará terriblemente insoportable y lento...."

B. (2) que/ a problemas futuros de de problemas de identidad y que puede afectarle de gran manera al niño en años posteriores además $e h$ creemos que los padres eh no deben disponer de la vida de de del ser que está por nacer por lo tanto los padres no no

Tanto en A como en B, la partícula cumple la función de agregar información. Asimismo, podemos señalar cómo en ambos casos se marca una pausa posterior a la partícula. En A, se representa ortográficamente mediante el uso de la coma, mientras que en $\mathrm{B}$, esta pausa se realiza por medio de "eh".

Para B, se añaden dos funciones:

-Retarda la continuación del discurso; en este ejemplo, después de la partícula se hace una pausa larga (duración mayor a un segundo):
(3) cómo está el conocimiento de religión// de Biblia de la persona// y aparte de eso le estamos metiendo un poco más de evangelización con las cuatro verdades espirituales/ y además// le llevamos un mensaje evangelístico a la persona dentro de la misma encuesta para que él

- Contribuye a señalar el inicio de un nuevo tema; se refuerza lo que menciona en la intervención anterior a la aparición de la partícula y se agrega el tema nuevo ("infertilidad como enfermedad y lo que la produce"):

(4) afectado su matrimonio y saber que existe una técnica que puede darles esa alegría de poder tener un niño

Estudiante 4: bueno estee además de que o sea como dijo la compañera antes la infertilidad sí es sí es una enfermedad/ hay una cosa que se llama fibrosis quística que le

\section{Entonces}

\section{Conector consecutivo}

A. (5) "Es q le amarraron un tarro d pintura blanca a la cola $d$ un burro y le pegaron una nalgada! Tonces [entonces] si las medidas quedaron mal, es culpa del burro"

B. (6) malas/ dice: y yo le voy a buscar una bien buena dice

Hombre: //tonces el viernes tengo/ el viernes tengo que darle la plata $\S$

Tanto A como B evidencian el uso de la partícula entonces como conector consecutivo. Cabe señalar que, en el caso de A, el hablante emplea una escritura apegada a la pronunciación de esta partícula propia del discurso oral informal. Dicha escritura (tonces) concuerda con la representación gráfica de la misma partícula en B. Podríamos plantear que, en el caso de A, parte de la adecuación del texto a un registro más informal implica retratar la pronunciación habitual en contextos informales.

Se identifica que, tanto para lo oral como para lo escrito, hay variantes en la escritura y la pronunciación, a saber: para ambos corpus: tonces; para el escrito: tons; para el oral: toes, 'ntoes, entoes.

Por otra parte, otras funciones en la oralidad son: 
- Llama la atención de los hablantes:

(7) Mujer 3: mjm

Mujer 2: ¿y entonces qué?

Mujer 3: que el mae es un inútil o que no sabe hacer nada

- Comprobar el contacto entre los hablantes:

(8) Mujer 2: eh ientonces?

Mujer 3: o sea (RISAS)

\section{O sea}

\section{Reformulador explicativo}

A.(9) "como si no fuera suficiente el que te rebajan mensualmente, no descansan la gripa y terminas con colitis y gastritis, o sea, no tenés derecho a la salud..."

B. (10) igual paja o sea cuántas cuántas mujeres no tienen relaciones una noche y pueden quedar embarazadas o// o sea// por Dios es lo mismo o sea puede ser que o que que estén casadas y se divorcien y al final el señor el señor se desentienda o sea se separen y

Los ejemplos A y B muestran el uso correspondiente a la reformulación explicativa, con la cual se aclara lo que se ha querido decir con otro miembro anterior. Se identifica el uso de pausas en ambos, en A se marca ortográficamente y en $\mathrm{B}$, en la segunda línea, observamos la aparición de la partícula entre pausas largas (de más de un segundo, // o sea//).

Se identifican las siguientes variantes en el corpus escrito: osea, y en el oral: 'sea.

Entre otras funciones que cumple en la oralidad, están:

\section{- $\quad$ Llama la atención del interlocutor:}

(11) Mujer 1: ¿cuántos años tiene tu mamá?

Mujer 3: tendría 36// ya se murió entonces este...// diay sí// o sea te imaginás// mi mamá así como...// mirá yo me puedo imaginar diciendo diay no Mariela yo creo que otra vez ya yo i

- Contribuye a introducir información nueva:

(12) verdad// o mantiene la creencia de que// de que no verdad// virgen hasta el...

Mujer 3: pues o sea diay sí está bien/ digo yo ' sea que que se casen la digamos// pero diay mae " sea uno no siempre va a seguir esos estereotipos de que iyo me quiero casar!

\section{Más bien}

\section{Reformulador de rectificación}

A. (13) "Qué porquería de noticias tenemos a veces... más bien me pregunto: ¿tenemos noticieros?"

B. (14) Mujer 1: ahí ahí lo tengo yo en la oficina (ininteligible)

Hombre 1: ah ves/ es que a este le faltó más bien fotocopiarlo

Más bien corrige un enunciado previo al introducido por la partícula. En ambos ejemplos, esta ocupa una posición parentética, es decir, se ubica en medio del enunciado. En el caso de A, se establece una pausa previa, que es indicada en el texto por medio de los puntos suspensivos al final del primer enunciado; por su parte, en B, no se registran pausas asociadas a su uso.

Para el caso de la oralidad, se consignó, además, el siguiente empleo:

- Introduce una apreciación (puede ser contraria a lo enunciado por otro hablante)

(15) Secretaria 1: ah muchas gracias Pedro/ ya eso es más que suficiente

Hombre 5: y ahí aprendés más bien

Secretaria 1: sí también diay sí/ uno de lo que no conoce hay que aprender

En cuanto a la posición que puede ocupar dentro del discurso, para A, solo se registró en posición parentética, mientras que, en $\mathrm{B}$, aparece en todas las posiciones.

\section{Claro}

Operador discursivo de refuerzo argumentativo

A. (16)“Me fascina el clima en estos días, full sol, como para andar de fiesta permanente. Claro que así cuesta mucho sentarse frente a la compu y concentrarse en el brete, pero en fin"

B. (17) Mujer 1: un parcito de medias así/ Néstor/ un parcito de medias así/ mil doscientos/ mil quinientos pesos/ para el súper vestido/// claro lindísimas/ de esas bonitas bonitas verdad Hombre: ¡ay Dios! 
En ambos casos, cumplen las funciones señaladas para este tipo de partículas, es decir, refuerza como argumento el miembro del discurso en el que se encuentra frente a otros posibles argumentos.

Asimismo, los dos corpus cuentan con ejemplos de uso con alargamiento vocálico, lo que representa que se enfatiza la respuesta; en estos contextos, siguiendo a Solano Rojas (1990: 14), podría tomar un valor adversativo:

A. (18) “.... jajaja mae, fui a un chante a ver las herramientas de tortura de la Inquisiciòn...y tenìan la vara donde ponìn a las acusadas de brujerìa... claaaro... que salì corriendo de ahi no fuera que me dejaran JAJAJA"

B. (19) Racu... el veneno está donde ellos no pasan Hombre: ¡sí claaro! no va a pasar por aquí por la basura ija! ahí hay uno

Mujer 1: ¡ay por el amor de Dios/ hacé caso!

Aunque no estamos haciendo referencia a la frecuencia de uso, en los corpus utilizados aparece cinco veces, en el escrito y el oral, respectivamente, lo que indica que no es común en las conversaciones informales del habla costarricense.

\section{De hecho}

Operador de refuerzo argumentativo

A. (20) "Esa población es de hecho muy famosa, tiene incluso un equipo que compite en la $2^{\circ}$ División del Futbol Profesional."

B. (21) sistema nervioso cuando el embrión tiene antes como un pre-embrión que no siente dolor no tiene nada (ininteligible) de la realidad jurídica// de hecho// se considera como que esta concepción de que de que todo óvulo fecundado ya es un ser humano más que todo son

La función de esta partícula es reforzar el argumento que la antecede, tal como se aprecia en los ejemplos anteriores. Con respecto a la posición que ocupan, en el caso de A, se registró en posición parentética e inicial, mientras que, en $\mathrm{B}$, solo en posición final e inicial. Por otra parte, en A, la partícula aparece integrada al enunciado, sin pausas visibles, mientras que, en $\mathrm{B}$, aparece en medio de dos pausas cortas.

\section{En realidad}

Operador de refuerzo argumentativo

A. (22) "En realidad, es una decision personal y NO DEBE DOLER!!!"

B. (23) concepción de que de que todo óvulo fecundado ya es un ser humano más que todo son intereses tradicionales e incluso se les puede llegar a llamar religiosos pero en realidad no es lo que está probado científicamente

Como se observa, en los dos ejemplos cumple la función de reforzar como argumento el miembro del discurso en el que se encuentra frente a otros posibles argumentos, explícitos o implícitos. Igualmente, como se indica en la Gramática Descriptiva de la Lengua Española (1999: 4140), presenta el miembro del discurso que lo incluye como una "realidad", la cual se distingue de otro argumento que se muestra como una "apariencia"; así, el primer argumento tiene más fuerza para conducir a unas conclusiones determinadas que el otro argumento.

\section{Al final}

\section{Reformulador de recapitulación}

A. (24) "te rebajan dinero como si no fuera suficiente el que te rebajan mensualmente, no descansan la gripa y terminas con colitis y gastritis, o sea, no tenés derecho a la salud... plofffff!!! Al final te obligan a ir a trabajar y propagas a todo el personal"

\section{B. (25) Mujer 1: [iqué horror!]}

Mujer 2: [ve doña Gloria]// entonces al final él no comió nada// quedó con hambre más bien Mujer 1: [sí sí sí]

Como reformulador de recapitulación, introduce una conclusión que se desprende de los enunciados previos. Puede aparecer al inicio, como se muestra en los ejemplos anteriores, o bien, en posición parentética. Con respecto a las pausas, en el discurso oral no se registran, mientras que en el discurso de Facebook, en la mayoría de los casos, aparece en medio de ellas.

Por otra parte, para el discurso oral, también se presenta el siguiente uso:

- $\quad$ Introduce una consecuencia de la primera parte del enunciado, tal como se observa en el siguiente ejemplo: 
(26) ahí a que haya nacido una chiquita un (ininteligible) pudo haber sido usado el mismo este esperma y se fecundó y se tuvo otro este otro joven que al final va a llegar a casarse hay problemas consanguíneos o se puede llegar a dar con este anonimato este

\section{Bueno}

Reactivo de desacuerdo, comentador, operador de refuerzo argumentativo.

A. (27) -"Bueno mae, eso es por irritaciones en las vias respiratorias y no es normal!"

(28) "No se puede creer ...Bueno, en realidad sí"

(29) "a mi me gusta más que me hablen de vos... pero es de confianza y respeto, y bueno Ale no le tiene confianza a nadie."

En la oralidad, cumple las funciones características del operador de formulación y marcador conversacional de control de contacto.

B. (30) Estudiante 2: [no nosotros consideramos que la fertilización in vitro no viola eh la la el derecho a la vida porque bueno como lo dijimos antes este bueno es una cuestión científica de que se está antes aunque se desarrolle el sistema nervioso entonces bueno 1 ... o sea

(31) Mujer 2: sí

Mujer 3: este/ ah bueno///pero también la persona que sí le interesa tanto lo físico diay mae/o sea nada más/porque vos no le vas a quitar ese placer/o sea/y tiene derecho a

Además, podemos observar que, tanto en la oralidad como en el discurso escrito, es posible encontrar usos similares, como en el reactivo de desacuerdo:

A. (32) - La mayoría de la gente aquí en mi casa respira con la jeta abierta...

-Bueno mae, eso es por irritaciones en las vias respiratorias y no es normal!

B. (33) Hombre 1: en esa iglesia hay gente en todo Hombre 2: ///bueno diay/pero bueno///volvamos Hombre 1: es que el problema fue que.../como que le ganaron una gorra

El reactivo de desacuerdo, como lo indican Calsamiglia y Tusón (1999), enfatiza la posición de desacuerdo del hablante ante lo que el interlocutor indica y bueno refuerza la imagen positiva del que habla y protege, al mismo tiempo, la imagen negativa del oyente (Martín Zorraquino y Portolés, 1999). A su vez, en lo oral, en su función de enfocador de la alteridad, introduce una réplica que implica un desacuerdo con el interlocutor y su uso atenúa este desacuerdo.

En el caso del comentador, consideramos que su función también es la confirmar un enunciado, como se realiza en la oralidad:

A. (34) - a ver, todos las que quieran contribuir con tarritos de pintura verde para tapar las medidas erróneas en la marcación de la cancha del Estadio Nacional... a ver... colaborando... siguen sin poder taparlas :P

- No se puede creer ...Bueno, en realidad sí

\section{B. (35) biblioteca mejor}

Entrevistador: si bueno usted tiene que venir/de lunes a viernes y hablar con la secretaria Informante 14: ajá

Esto debido a que entre sus significados está el de consentimiento, lo que explica los usos de corrección o autocorrección; con este se admite o previene un posible desacuerdo del interlocutor (Portolés, 2001).

\section{Diay}

Comentador, operador de refuerzo argumentativo y reactivo de desacuerdo.

A.(36) "diay acá ando jajajajaja....en el centro histórico wevón!!!”

(37)“justo a eso me refería, Marjorie.... era justo eso: que asociaron la formalidad que uno tiene, yai [diay] porque le estan pidiendo o dando trabajo, tons [entonces] yai [diay] uno responde asi formalito.... y cree que uno es viejisimo... tiene ud razón"

(38) "Ya me curé, no necesito que me vean! No importa, tiene que sacar cita. Ok a que hora tengo que venir? No sé, pregúntele a alguien. A quién? Diay, usted no vive aquí?"

Por su parte, en la oralidad, también se identificaron las funciones de comentador y de operador de refuerzo argumentativo, tal como se aprecia en los siguientes ejemplos: 
B. (39) Secretaria 2: el cheque de los asistentes / profesor cómo está

Hombre 1: cómo vas

Hombre 4: //diay digamos que bien/ tengo examen el viernes

Hombre 1: ajá pero un examen bonito obviamente

(40) Hombre 2: peor no lo vendió aquí pero yo a él es que el mae tiene a veces lleva varias series yo le juego el cero siete siempre entero diay güevón el mae me dejó el el hijueputa cero siete de la novecientos tres el treinta y nueve de la seiscientos diez y siete y el cincuenta y tres de la setecientos

Tanto en lo escrito como en lo oral, en su función de comentador, la partícula introduce un nuevo enunciado en las conversaciones. Específicamente, funciona como un marcador de apertura, de ahí que aparezca al inicio del enunciado que introduce, tal como se evidencia en los ejemplos consignados. Con respecto a su función de operador de refuerzo argumentativo, podríamos afirmar, tal como lo plantea Hernández (2012), que la forma "diay" refuerza el enunciado que introduce con una carga actitudinal extra, es decir, posee un valor modal dentro del discurso. En relación con este último uso, Benavides (2014) añade que, además de introducir un nuevo comentario, la partícula cumple una función de marcador de control de contacto, o sea, refuerza su argumento ante los interlocutores.

Por otra parte, el uso como reactivo de desacuerdo, documentado por Pérez (2014), no aparece registrado para el discurso oral; sin embargo, Benavides (2014) reconoce otras funciones específicas asociadas a "diay". Así entonces, en su clasificación, esta partícula aparece como conector consecutivo, reformulador explicativo, reemplazo de pausa y reformulador recapitulativo. A continuación, ilustramos algunos de estos usos:

(41) Mujer 2: [y no y no nos entendimos en la cama]/ entonces diay [fracasa todo el matrimonio]

Mujer 1: [o sea diay] entonces si hay algo ahí que que anda ahí/ rondando acerca de de////di// de que las relaciones/ las relaciones en general mantienen/ se mantienen por el sexo/

Acerca de las variantes fonéticas y de escritura, se registró la forma yai en el corpus escrito, probablemente como una reproducción de la manera en que se suele pronunciar esta partícula en el habla informal, a pesar de que para el corpus oral solo se identificó la variante $d i$.

\section{Es que}

\section{Reformulador explicativo}

A. (42) -"no soy problematica! Es que yo no se que se cree ese enano"

B. (43) Mujer 3: y y diay si te toca un mae que es un inútil y vos casada con él

Mujer 2: por eso/// es que digamos el sexo sí es parte muy importante de cualquier relación§

En ambos ejemplos, se presenta como una nueva formulación que aclara y explica lo que se ha querido decir; en el caso del ejemplo $\mathrm{B}$, del discurso oral, se refuerza con el también reformulador explicativo digamos.

Por otra parte, en el corpus correspondiente a la oralidad, se identificaron en es que las funciones de conector, específicamente conector aditivo y conector consecutivo:

B. (44) Hombre: [tan mala]

Mujer 1: estos son más buenas // y es que estas chunchas tienen repuesto

Hombre: sí/ lo que se les va es la resistencia§

(45) Mujer 2: no no es que uno se (ininteligible)§ Mujer 3: §es que es que también así como dicen es problema de mi signo zodiacal entonces..

Se identificaron las siguientes variantes: $e^{\prime}$ que (aspiración de s), en la oralidad, y es $q$, para el corpus escrito.

\section{Anotaciones finales}

A manera de síntesis, señalamos aquí las consideraciones finales acerca de esta comparación de partículas discursivas elaboradas en el discurso oral y en el escrito en Facebook.

Se evidencia que ciertas partículas, en ambos tipos de discurso, mantienen sus características y funciones. Esto ocurre con los clasificados como conectores (además, entonces), 
reformuladores (o sea, más bien, al final, es que) y los operadores discursivos (claro, de hecho, en realidad); no obstante, en la oralidad, a partir del análisis de los rasgos suprasegmentales y el contexto es posible encontrar funciones que pertenecen a otras categorías (no fue estudiado a profundidad en este artículo, pero en los ejemplos sobre bueno, diay encontramos mayor diversidad de funciones).

Referente a las pausas que suelen acompañar a las partículas estudiadas, hemos identificado que estas constituyen un elemento importante para definir el valor semántico de estas y su función discursiva. Además, dichas pausas son codificadas según las posibilidades que ofrece cada modalidad. Específicamente, en el discurso de Facebook, los hablantes recurren a las comas o a los puntos suspensivos para reemplazar las pausas propias de la oralidad, aunque su empleo no sea adecuado en cada caso.

Quizá uno de los rasgos de la oralidad más evidentes en el discurso de Facebook sea la correspondencia entre la pronunciación y la escritura. En este tipo de escritura, los hablantes buscan retratar el estilo de habla informal, tal como se evidenció con las formas entonces (tons, tonces...) o diay (yai, di). No obstante, en la escritura de Facebook, son frecuentes otros recursos que afectan la forma, pero que se encuentran más vinculados a procedimientos de abreviación, típicos de la ciberhabla, por ejemplo, es $q$ en vez de es que o $x q$ en lugar de porque.

Observamos que, más que "errores" ortográficos, las adaptaciones en la escritura para representar la pronunciación típica de la oralidad evidencian la existencia de una conciencia fonológica de la lengua. Este conocimiento se logra plasmar en el texto gracias a la creatividad lingüística del hablante.

Asimismo, no solo se adaptan los rasgos fonéticos a la escritura, sino también aspectos suprasegmentales. Tal como se pudo apreciar en algunos ejemplos utilizados en el análisis (B.4, A.18, B.19), muchos de los alargamientos vocálicos están relacionados también con patrones entonativos que permiten determinar el significado y función de una partícula específica, con respecto a otros usos que la misma forma puede presentar. Entre ellos tenemos también, en el discurso oral, que la contextualización visual es determinante para otorgar sentido al enunciado; lo cual se resuelve, en el discurso escrito en Facebook, recurriendo a los emoticones (como :P, XD, ;), :-O, :S) para sustituir las expresiones faciales y evitar malentendidos; ciertos de estos símbolos refuerzan el significado de las partículas, como ocurre con bueno-:P. Este último punto, no se analiza en el presente artículo, pero consideramos que merece ser estudiado con mayor profundidad en otros estudios sobre el lenguaje en Internet.

Finalmente, a partir de lo observado en esta comparación, consideramos que la escritura en Internet, en particular en una red social como Facebook, corresponde a una modalidad discursiva particular, al parecer, más cercana a la oral pero con medios particulares para comunicar el mensaje.

\section{Notas}

1. Estos rasgos, a su vez, contienen los siguientes aspectos específicos:

Prosódicos: unidad tónica, aislado por pausas (precedido o seguido por estas).

Morfosintácticos: unidad invariable, posición extrapredicativa, invariabilidad flexiva, puede omitirse sin afectar la relación con enunciados contiguos, contenido proposicional, relación enunciativa, aparece solo en turno de palabra.

Semánticos: significado relacional (remite a contenidos previos o posteriores), papel metadiscursivo, apelativo; no contribuye a las condiciones de verdad de la proposición.

Pragmáticos: significado procedimental, instrucciones semánticas.

2. Portolés denomina a este grupo "operadores discursivos"; y agrega el "operador de formulación", el cual se encuentra representado por bueno (formulación que transmite satisfactoriamente la intención comunicativa del hablante). Finalmente, a los "marcadores conversacionales" los designa "marcadores de control de contacto" (manifiestan la relación entre los participantes de la conversación). 


\section{Bibliografía}

Beaugrande, R. y Dressler, W. 1997. Introducción a la lingüística del texto. Barcelona: Editorial Ariel.

Benavides, M. 2014. Los marcadores discursivos en el español informal en Costa Rica: una propuesta de diccionario. Tesis de maestría en Lingüística. Universidad de Costa Rica.

Bosque, I. y V. Demonte (eds.). 1999. Gramática descriptiva de la lengua española. Madrid: España.

Briz, A., S. Pons y J. Portolés (coords.). 2008. Diccionario de partículas discursivas del español (DPDE). En línea: http://www. dpde.es/.

Briz, A. y Val.Es.Co (2000). ¿Cómo se comenta un texto coloquial? Barcelona: Editorial Ariel, S. A.

Calsamiglia, H. y Tusón (1999). Las cosas del decir. Manual de análisis del discurso. Barcelona: Ariel.

Castro Corrales, A. A. (2004). Marcadores argumentativos y contraargumentativos en el discurso parlamentario costarricense: Ley para el mejoramiento de servicios públicos de electricidad telecomunicaciones y de la participación del Estado, N. ${ }^{o}$ 13.873. Universidad de Costa Rica: Tesis de Licenciatura.

Garcés Gómez, M. 2008. La organización del discurso: marcadores de ordenación y reformulación. Madrid/Frankfurt: Iberoamericana Vervuert.

Hernández, M. (2012). Usos y funciones de i(diay) en Costa Rica. Káñina, Revista de Artes y Letras, 36 (Especial): 101-110.

Pérez, D. 2014. Mecanismos de cohesión textual en las conversaciones de Facebook. Tesis de maestría en Lingüística. Universidad de Costa Rica.

Porto Dapena, J.-Á. 2002. Manual de técnica lexicográfica. Madrid: Arco Libros, S. A.

Portolés, J. 2001. Marcadores del discurso. $2^{\mathrm{a}}$ ed. Barcelona: Ariel.

Sánchez, C. (2005). Los conectores discursivos: su empleo en redacciones de estudiantes universitarios costarricenses. Revista de Filología y Lingüística de la Universidad de Costa Rica, 31(2): 169-199.

Solano Rojas, Y. (1989). Los conectores pragmáticos en el habla culta costarricense. Revista de Filología y Lingüística de la Universidad de Costa Rica, 15(2): 143-154.

Solano Rojas, Y. (1990). Los nexos en el habla culta costarricense. Tesis para optar por el grado de Doctorado en Letras con especialidad en Lingüística Hispánica, México.

Solano Rojas, Y. (1991). Las formas nexuales adversativas en el habla culta costarricense. Revista de Filología y Lingüística de la Universidad de Costa Rica, 17(1-2): 219-234.

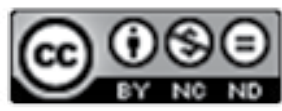

Este obra está bajo una licencia de Creative Commons

Reconocimiento-NoComercial-SinObraDerivada 4.0 Internacional. 